\title{
Fatigue crack growth assessment method subject to model uncertainty
}

\section{Yan-Hui Lin ${ }^{\mathrm{a}, \mathrm{b}}$}

linyanhui@buaa.edu.cn

Ze-Qi Dinga, b

Enrico Zio ${ }^{c,}$

aschool of Reliability and Systems Engineering, Beihang University, Beijing, China

bScience and Technology on Reliability and Environmental Engineering Laboratory, Beihang University, Beijing, China

${ }^{\mathbf{c} E n e r g y ~ D e p a r t m e n t, ~ P o l i t e c n i c o ~ d i ~ M i l a n o, ~ M i l a n, ~ I t a l y ~}$

dMines ParisTech, PSL University, Centre for Rresearch on Risk and Crises (CRC), Sophia-Antipolis, France

\section{Abstract}

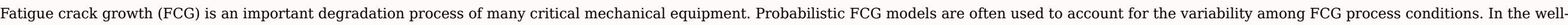

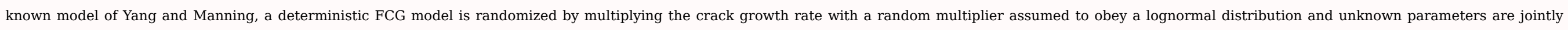

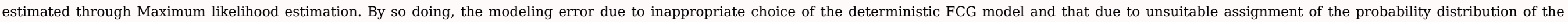

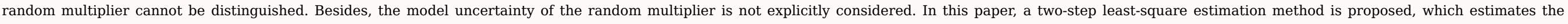

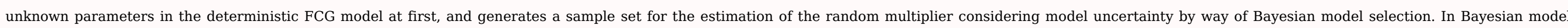

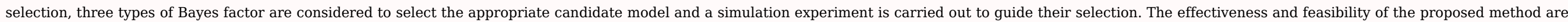
illustrated through two case studies using the real FCG datasets.

Keywords: Fatigue crack growth analysis; Model uncertainty; Yang and Manning's probabilistic FCG model; Two-step least-square estimation; Bayesian model selection

\section{Introduction}

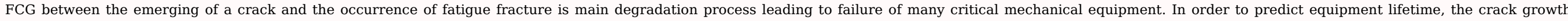

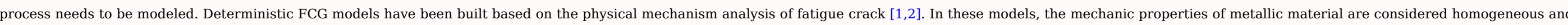

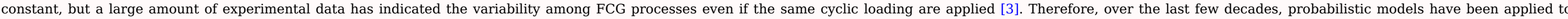
incorporate randomness in the FCG process [4].

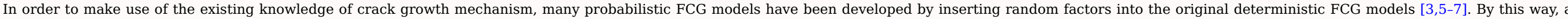

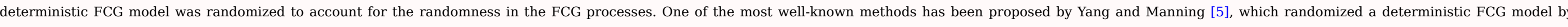

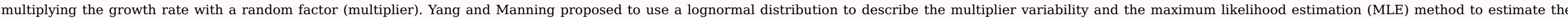
unknown parameters in the deterministic FCG model and in the lognormal distribution of the random multiplier [5].

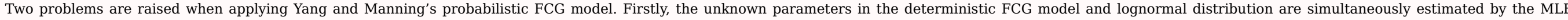

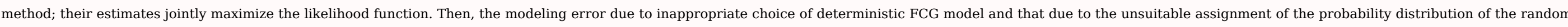

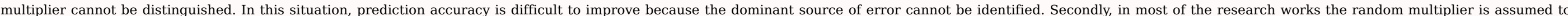




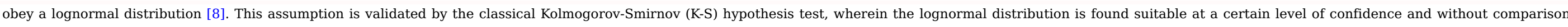
with other candidate distribution. Therefore, the uncertainty in determining the best distribution model should be considered.

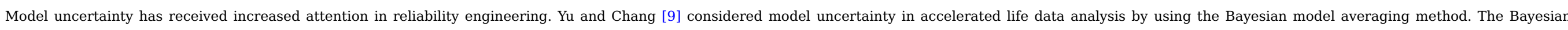

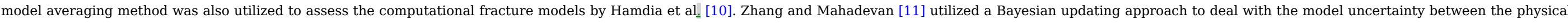

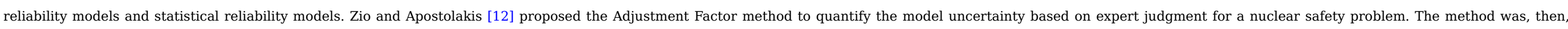

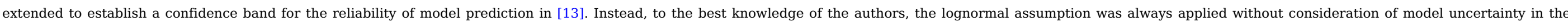
application of Yang and Manning's model [3,14,15].

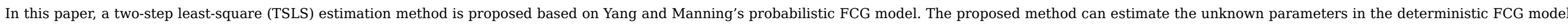

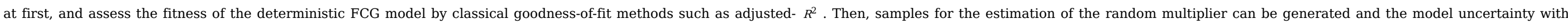

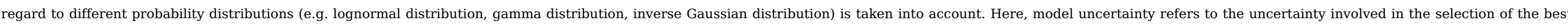

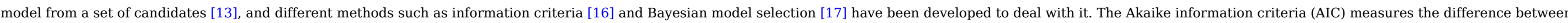

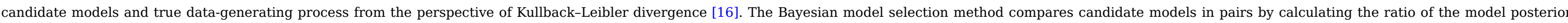

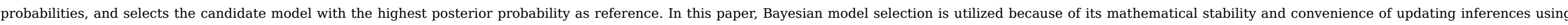

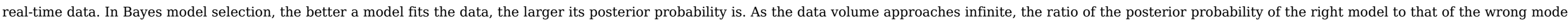

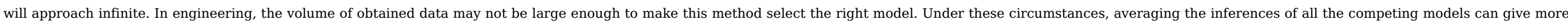
comprehensive results. In [18], the Bayesian model selection method is able to achieve better simulating results compared with AIC for degradation data analysis.

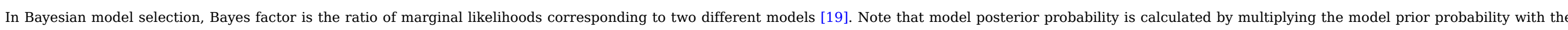

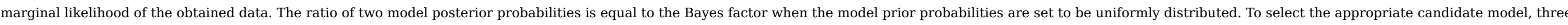

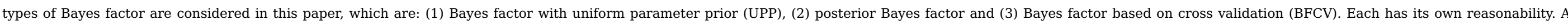
simulation experiment is carried out to compare their performance. According to the results, the BFCV is recommended.

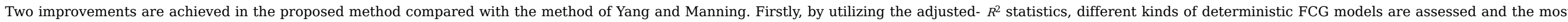

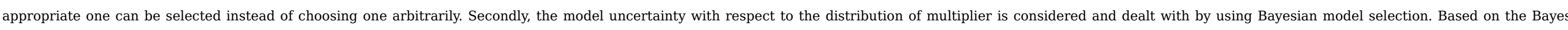

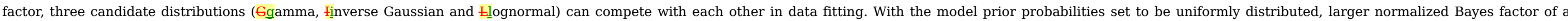

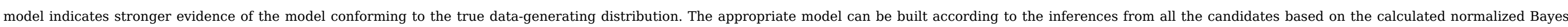
factors, which are defined as model weights [20,21]. In this way, the assumption of the lognormal distribution in the method of Yang and Manning can be relaxed.

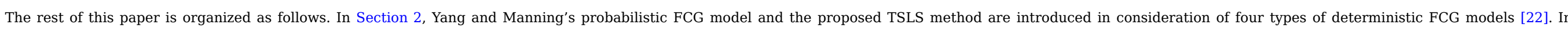

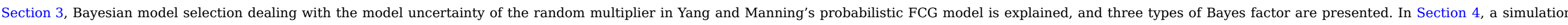

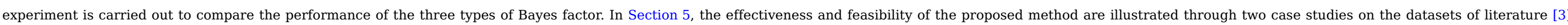
and [4]. Section 6 concludes the work.

\section{TSLS estimation method based on the Yang and Manning's probabilistic model}

\subsection{Yang and Manning's probabilistic model}

To consider the randomness in the fatigue crack growth process, Yang and Manning [5] proposed a very general formula frame to randomize deterministic FCG models as follows:

$$
\frac{d a(t)}{d t}=G\left(\Delta K, R, K_{\max }, S, a(t)\right) \cdot X(a(t))
$$

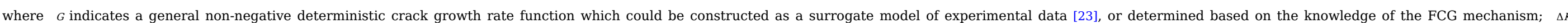




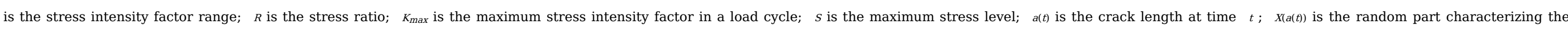

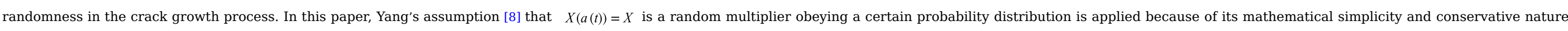
[24].

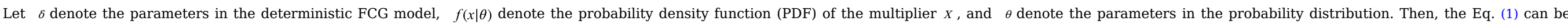
reformulated as:

$\frac{d a(t)}{d t}=G(a(t), \delta) \cdot X$

$X f(x \mid \theta) X \sim f(x \mid \theta)$

After the logarithm is taken on both sides of the Eq. (2), we can obtain that

$\log \left(\frac{d a(t)}{d t}\right)=\log (G(a(t), \delta))+Z$

where $Z=\log (X)$.

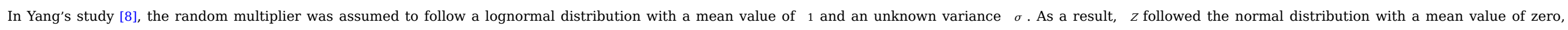
$Z N(0, \sigma) Z \sim N(0, \sigma)$, and

$\log \left(\frac{d a(t)}{d t}\right) N(\log (G(a(t), \delta)), \sigma) \log \left(\frac{d a(t)}{d t}\right) \sim N(\log (G(a(t), \delta)), \sigma)$

The PDF of crack growth rate is

$$
f_{\text {normal }}\left[\log \left(\frac{d a(t)}{d t}\right)\right]=\frac{1}{2 \pi \sigma} \exp \left[-\frac{\left(\log \left(\frac{d a(t)}{d t}\right)-\log (G(a(t), \delta))\right)^{2}}{2 \sigma^{2}}\right]
$$

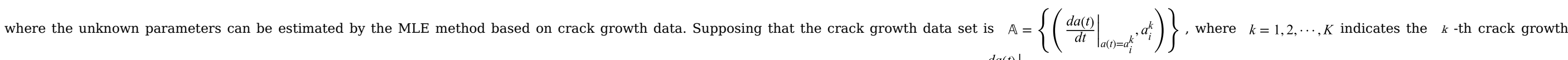

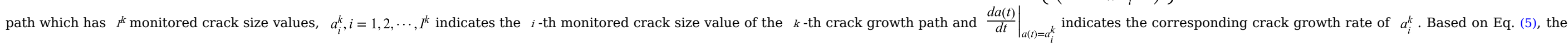
maximum likelihood estimators can be obtained as

$$
(\widehat{\delta}, \hat{\sigma})=\arg \max \left(\prod_{k=1}^{K} \prod_{i=1}^{I^{k}} f_{\text {normal }}\left(\log \left(\left.\frac{d a(t)}{d t}\right|_{a(t)=a_{i}^{k}}\right)\right)\right)
$$

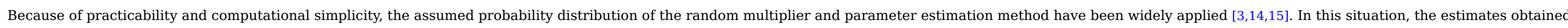

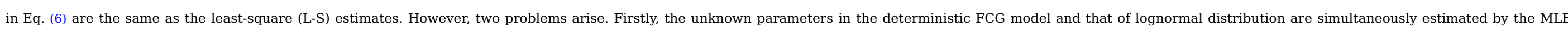

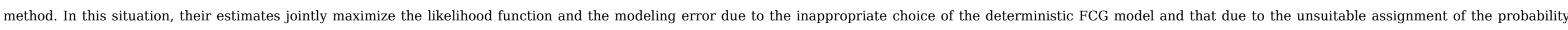

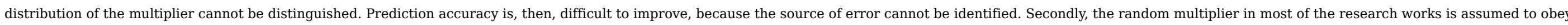

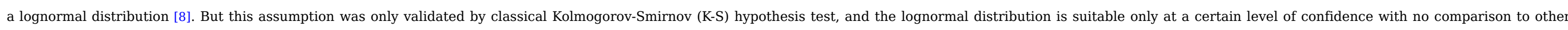
candidate distributions.

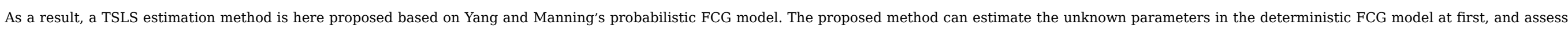

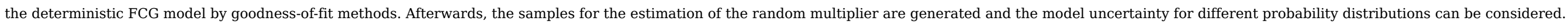




\subsection{TSLS estimation method}

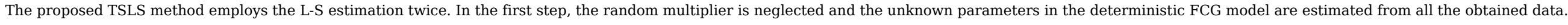

$\widehat{\delta}_{L-S}=\arg \min _{\delta}\left(\sum_{k=1}^{K} \sum_{i=1}^{I}\left(\log \left(\left.\frac{d a(t)}{d t}\right|_{a(t)=a_{i}^{k}}\right)-\log \left(G\left(a_{i}^{k}, \delta\right)\right)\right)^{2}\right)$

Annotations:

A1. please add a footnote ' $\mathrm{k}$ ' to the upper right corner of letter 'I'

In the second step, the random multiplier of each crack growth path is estimated as

$\widehat{z}_{k}=\arg \min _{z}\left(\sum_{i=1}^{I}\left(\log \left(\left.\frac{d a(t)}{d t}\right|_{a(t)=a_{i}^{k}}\right)-\log \left(G\left(a_{i}^{k}, \widehat{\delta}_{L-S}\right)\right)-z\right)^{2}\right), k=1,2, \cdots, K$

$\widehat{x}_{k}=\exp \left(\widehat{z}_{k}\right), k=1,2, \cdots, K$

where the $\hat{x}_{k}$ is the estimate of the random multiplier for the $k$-th crack growth path.

\section{Annotations:}

A1. please add a footnote ' $k$ ' to the upper right corner of letter 'I'

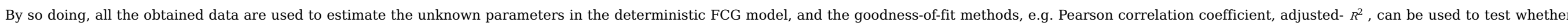

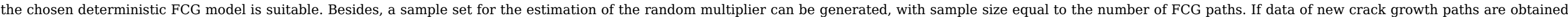

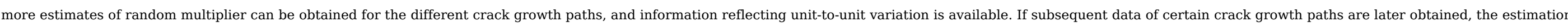
accuracy of the corresponding random multipliers can be further improved.

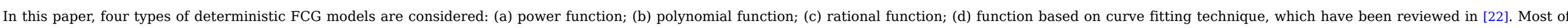

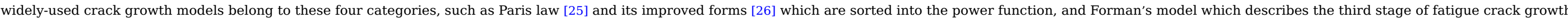
[27] can be sorted into the rational function. Their detailed formulas are as follows

Power function

$\frac{d a(t)}{d t}=X \cdot Q[a(t)]^{b}$

Polynomial function

$\frac{d a(t)}{d t}=X \cdot\left\{p+q \cdot a(t)+r \cdot[a(t)]^{2}\right\}$

Rational function

$\frac{d a(t)}{d t}=X \cdot \frac{Q_{1}[a(t)]^{b}}{Q_{2}-Q_{3} \sqrt{ } a(t)}$

Function based on curve fitting technique

$\frac{d a(t)}{d t}=X \cdot \frac{1}{Q_{1}[a(t)]^{b}+Q_{2}}$

The parameters which need to be estimated are $Q, b$ in power function, $p, q, r$ in polynomial function, $Q_{1}, Q_{2}, Q_{3}, b$ in rational function, and $Q_{1}, Q_{2}, b$ in function based on curve fitting technique. By replacing 
Power function:

\begin{tabular}{|c|c|c|c|c|c|c|c|c|}
\hline$\left(\widehat{Q}_{L-S}, \hat{b}_{L-S}\right)=\arg \min _{(Q, b)}$ & $\sum_{k=1}^{K} \sum_{i=1}^{I}\{\log$ & {$\left[\left.\frac{d a(t)}{d t}\right|_{a(t)=a_{i}^{k}}\right]$} & $\left.-b \cdot \log \left(d_{i}^{j}\right)+\log (Q)\right\}$ & \}$^{2} \mid\left(\hat{Q}_{L-S}, \hat{b}_{L-S}\right)$ & )$=\arg \min _{(,, b)}\left(\sum_{k=1}^{K} \sum_{i=1}^{l^{k}}\{\log \mid\right.$ & {$\left[\left.\frac{d a(t)}{d t}\right|_{a(t)=a_{i}^{k}}\right]$} & ]$\left.-b \cdot \log \left(a_{i}^{k}\right)-\log (Q)\right\}^{2} \mid$ & \\
\hline$\widehat{z}_{k}=\arg \min _{z}\left(\sum_{i=1}^{I}\{\log )\right.$ & {$\left[\left.\frac{d a(t)}{d t}\right|_{a(t)=a_{i}^{k}}\right.$} & $-\hat{b}_{L-S} \log$ & $\left.\left.d_{i}^{j}\right)+\log \left(\hat{Q}_{L-S}\right)-z\right\}$ & $, k=1,2, \cdots, K$ & $\hat{z}_{k}=\arg \min _{z}\left(\sum_{i=1}^{l^{k}}\{\log [\right.$ & {$\left[\left.\frac{d a(t)}{d t}\right|_{a(t)=a_{i}^{k}}\right]$} & $-\hat{b}_{L-S} \log \left(a_{i}^{k}\right)-\log (\hat{c}$ & $, k=1,2, \cdots, K$ \\
\hline
\end{tabular}

Polynomial function:

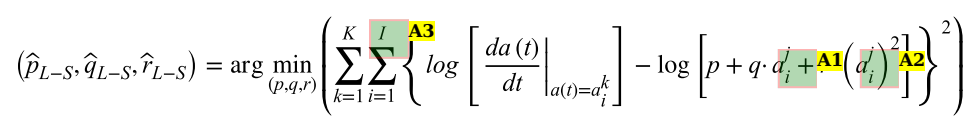

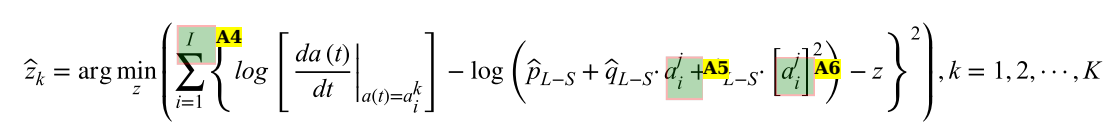

\section{Annotations:}

A1. please replace the upper right footnote 'j' by letter ' $k$ '

A2. please replace the upper right footnote ' $j$ ' by letter ' $k$ '

A3. please add a footnote ' $\mathrm{k}$ ' to the upper right corner of letter 'I'

A4. please add a footnote ' $k$ ' to the upper right corner of letter 'I'

A5. please replace the upper right footnote ' $j$ ' by letter ' $\mathrm{k}$ '

A6. please replace the upper right footnote ' $\mathrm{j}$ ' by letter ' $\mathrm{k}$ '

Rational function:

$$
\begin{aligned}
& \left.\left(\widehat{Q}_{1 L-S}, \widehat{Q}_{2 L-S}, \widehat{Q}_{3 L-S}, \hat{b}_{L-S}\right)=\arg \min _{\left(Q_{1}, Q_{2}, Q_{3}, b\right)}\left(\sum_{k=1}^{K} \sum_{i=1}^{I} \int^{\mathbf{A} 3} \log \left[\left.\frac{d a(t)}{d t}\right|_{a(t)=a_{i}^{k}}\right]+\log \left(Q_{2}-Q_{3} \sqrt{a_{i}^{j}}\right) \mathbf{A} \mathbf{2 b} \cdot \log \left(a_{i}^{j}\right) \mathbf{A} \mathbf{1} \log \left(Q_{1}\right)\right\}^{2}\right) \\
& \widehat{z}_{k}=\arg \min _{z}\left(\sum_{i=1}^{I}\left\{\log \left[\left.\frac{d a(t)}{d t}\right|_{a(t)=a_{i}^{k}}\right]+\log \left(\widehat{Q}_{2 L-S}-\widehat{Q}_{3 L-S} \sqrt{a_{i}^{j}}\right) \mathbf{A} 5 \widehat{b}_{L-S} \log \left(d_{i}^{j}\right) \mathbf{A 6} \log \left(\widehat{Q}_{1 L-S}\right)-z\right\}^{2}\right), k=1,2, \cdots, K
\end{aligned}
$$
Annotations:
A1. please replace the upper right footnote ' $j$ ' by letter ' $k$ '
A2. please replace the upper right footnote ' $j$ ' by letter ' $k$ '
A3. please add a footnote ' $\mathrm{k}$ ' to the upper right corner of letter 'I'
A4. please add a footnote ' $k$ ' to the upper right corner of letter 'I'
A5. please replace the upper right footnote ' 'j' by letter ' $k$ '
A6. please replace the upper right footnote ' $\mathrm{j}$ ' by letter ' $\mathrm{k}$ ' 


$$
\begin{aligned}
& \left(\widehat{Q}_{1 L-S}, \widehat{Q}_{2 L-S}, \widehat{b}_{L-S}\right)=\arg \min _{\left(Q_{1}, Q_{2}, b\right)}\left(\sum_{k=1}^{K} \sum_{i=1}^{I}\left\{\operatorname{Al}\left[\log \left[\left.\frac{d a(t)}{d t}\right|_{a(t)=a_{i}^{k}}\right]+\log \left[Q_{1}\left(a_{i}^{k}\right)^{b}+Q_{2}\right]\right\}^{2}\right)\right. \\
& \widehat{z}_{k}=\arg \min _{z}\left(\sum_{i=1}^{I}\left\{\log \left[\left.\frac{d a(t)}{d t}\right|_{a(t)=a_{i}^{k}}\right]+\log \left[\widehat{Q}_{1 L-S}\left(a_{i}^{k}\right)^{\hat{b}_{L-S}}+\widehat{Q}_{2 L-S}\right]-z\right\}^{2}\right), k=1,2, \cdots, K
\end{aligned}
$$

\section{Annotations:}

A1. please add a footnote ' $\mathrm{k}$ ' to the upper right corner of letter 'I'

A2. please add a footnote ' $\mathrm{k}$ ' to the upper right corner of letter ' $\mathrm{I}$ '

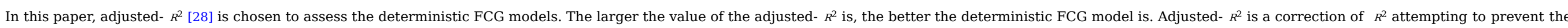
automatically and spuriously increasing of $R^{2}$ when extra parameters are added into the model. The adjusted- $R^{2}$ can be obtained as

adjusted $-R^{2}=1-\frac{\left(\sum_{k=1}^{K} I^{k}\right)-1}{\left(\sum_{k=1}^{K} I^{k}\right)-p-1} \cdot \frac{S S_{\text {res }}}{S S_{\text {tot }}}$

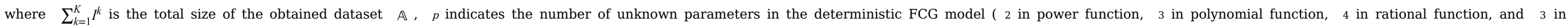
function based on curve fitting technique), $s S_{\text {tot }}$ denotes the total sum of squares which is obtained as

$$
S S_{t o t}=\sum_{k=1}^{K} \sum_{i=1}^{I}\left\{\operatorname{Al}\left[\log \left[\left.\frac{d a(t)}{d t}\right|_{a(t)=a_{i}^{k}}\right]-\frac{1}{\sum_{k=1}^{K} I^{k}} \sum_{k=1}^{K} \sum_{i=1}^{I^{k}}\left\{\log \left[\left.\frac{d a(t)}{d t}\right|_{a(t)=a_{i}^{k}}\right]\right\}\right\}^{2}\right.
$$

and $S S_{r e s}$ denotes the residual sum of squares which is obtained as

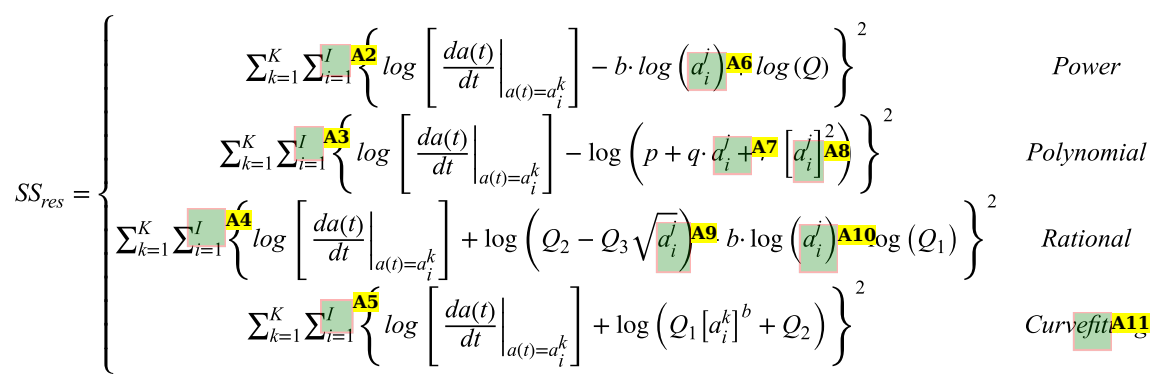

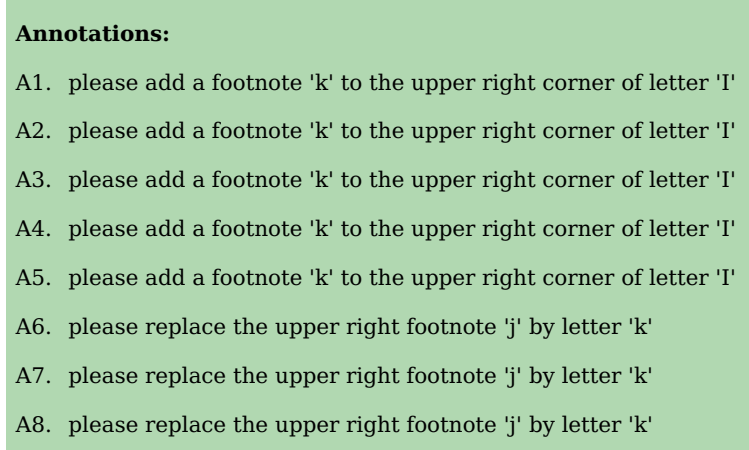

A1. please add a footnote ' $k$ ' to the upper right corner of letter 'I'

A2. please add a footnote ' $\mathrm{k}$ ' to the upper right corner of letter ' $\mathrm{I}$ '

A3. please add a footnote ' $k$ ' to the upper right corner of letter 'I'

A4. please add a footnote ' $\mathrm{k}$ ' to the upper right corner of letter ' $\mathrm{I}$ '

A5. please add a footnote ' $k$ ' to the upper right corner of letter 'I'

A6. please replace the upper right footnote ' $j$ ' by letter ' $k$ '

A7. please replace the upper right footnote ' $j$ ' by letter ' $k$ '

A8. please replace the upper right footnote ' $j$ ' by letter ' $k$ ' 


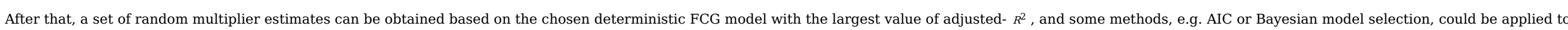

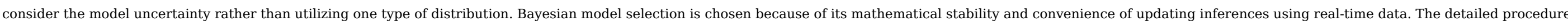
of Bayesian model selection is introduced in Section 3.

\section{Introduction and application of Bayesian model selection}

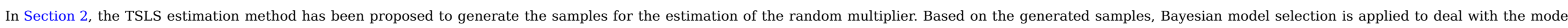

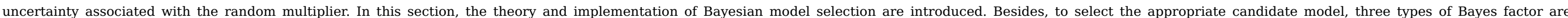
considered.

\subsection{The Bayesian model selection method}

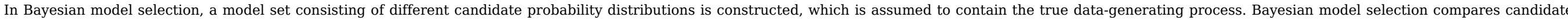

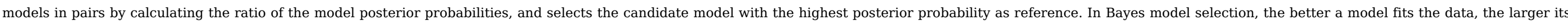

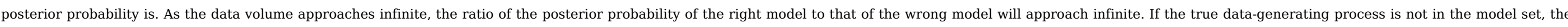

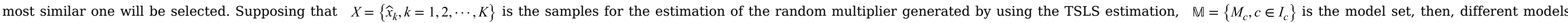
compete with each other by

$\frac{\text { posterior }\left(M_{p}\right)}{\text { posterior }\left(M_{q}\right)}=\frac{\operatorname{pr}\left(X \mid M_{p}\right) \cdot \operatorname{prior}\left(M_{p}\right)}{\operatorname{pr}\left(X \mid M_{q}\right) \cdot \operatorname{prior}\left(M_{q}\right)}, p, q \in I_{c}$

In Eq. (25), $\operatorname{prior}\left(M_{p}\right), p \in I_{c}$ is the model prior representing the prior evidence of the model $M_{p}$ before the data are obtained; $\operatorname{pr}\left(X \mid M_{p}\right), p \in I_{c}$ is the marginal likelihood calculated by

$\operatorname{pr}\left(X \mid M_{p}\right)=\int L\left(X \mid \theta_{p}, M_{p}\right) \operatorname{prior}\left(\theta_{p}\right) d \theta_{p}$

where $\operatorname{prior}\left(\theta_{c}\right)$ is the prior distribution of the parameter vector $\theta_{C}, L\left(X \mid \theta_{c}, M_{c}\right)$ is the likelihood function calculated by

$L\left(X \mid \theta_{p}, M_{p}\right)=\prod_{\hat{x}_{k} \in X} f\left(\hat{x}_{k} \mid \theta_{p}, M_{p}\right)$

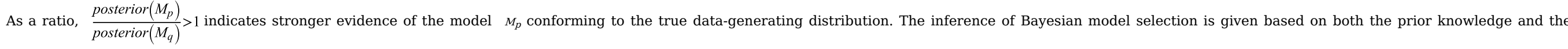

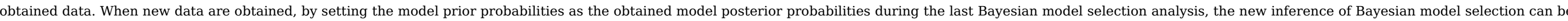

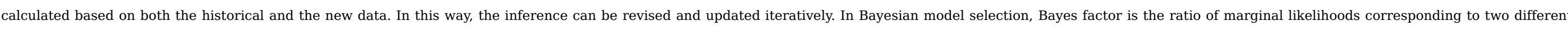
models [19]:

$B F_{p, q}=\frac{\operatorname{pr}\left(X \mid M_{p}\right)}{\operatorname{pr}\left(X \mid M_{q}\right)}, \quad p, q \in I_{c}$

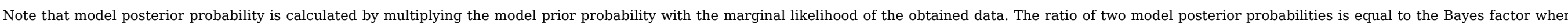

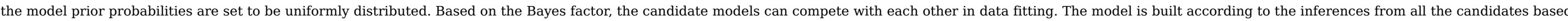

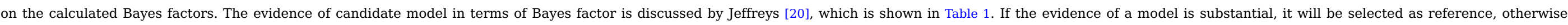


Table 1 Model building based on Bayes factor.

\begin{tabular}{|c|c|c|}
\hline $\mathrm{BF}_{\mathrm{p}, \mathrm{q}}<\frac{1}{3.162} B F_{p, q}<\frac{1}{3.162}$ & Substantial evidence against the model $\mathrm{M}_{\mathrm{p}} M_{p}$ & $\mathrm{M}_{\mathrm{p}} M_{p}$ is abandoned \\
\hline \begin{tabular}{|l|l|}
$\frac{1}{3.162}<\mathrm{BF}_{\mathrm{p}, \mathrm{<}}<3.162$ & $\frac{1}{3.162}<B F_{p, q}<3.162$ \\
\end{tabular} & insufficient evidence & Model $\mathrm{M}_{\mathrm{q}} M_{q}$ and $\mathrm{M}_{\mathrm{p}} M_{p}$ are averaged \\
\hline \begin{tabular}{|l|l|}
$\mathrm{BF}_{\mathrm{p}, \mathrm{q}}>3.162$ & $B F_{p, q}>3.162$ \\
\end{tabular} & Substantial evidence of the model $\mathbf{M}_{\mathrm{p}} M_{p}$ & $\mathrm{M}_{\mathrm{p}} M_{p}$ is selected \\
\hline
\end{tabular}

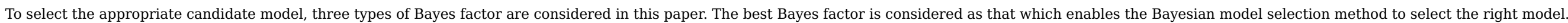

\subsection{Three types of Bayes factor}

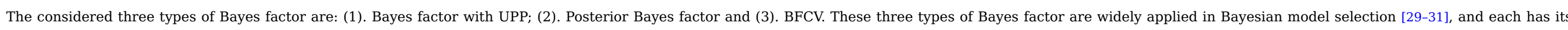
own reasonability.

\subsubsection{Bayes factor with UPP}

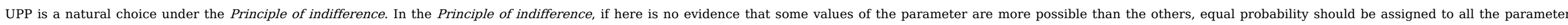
values. Therefore, a uniform distribution is considered as an objective choice before any data is obtained.

prior $_{\text {uniform }}(\theta) \propto 1$

and Eqs. (26) and (28) can be reformulated as

uni $-\operatorname{pr}\left(X \mid M_{p}\right)=\int L\left(X \mid \theta_{p}, M_{p}\right)$ prior $_{\text {uniform }}\left(\theta_{p}\right) d \theta_{p} \quad \mathbf{A 1}$

$u n i-B F_{p, q}=\frac{u n i-p r\left(X \mid M_{p}\right)}{u n i-p r\left(X \mid M_{q}\right)}, \quad p, q \in I_{c}$

Annotations:

A1. please add a ' $\mathrm{p} \in \mathrm{I} \_$c,' at the end of the formula

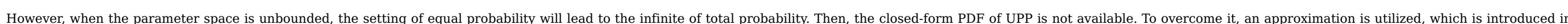
Section 3.3.3.

\subsubsection{Posterior Bayes factor}

The posterior Bayes factor [29] utilizes the Bayes theorem and all the obtained data to update the UPP first; then, the updated parameter distribution is used for the calculation of the Bayes factor, which is obtained as

$\operatorname{pos}-B F_{p, q}=\frac{\operatorname{pos}-\operatorname{pr}\left(X \mid M_{p}\right)}{\operatorname{pos}-\operatorname{pr}\left(X \mid M_{q}\right)}, \quad p, q \in I_{c}$

pos $-\operatorname{pr}\left(X \mid M_{p}\right)=\int L\left(X \mid \theta_{p}, M_{p}\right) \cdot \operatorname{posterior}\left(\theta_{p}\right) d \theta_{p} \mid \mathbf{A 1}$

where

posterior $\left(\theta_{p}\right) \propto L\left(X \mid \theta_{p}, M_{p}\right) \times$ prior $_{\text {uniform }}\left(\theta_{p}\right)$ 


\subsubsection{Bayes factor based on cross validation}

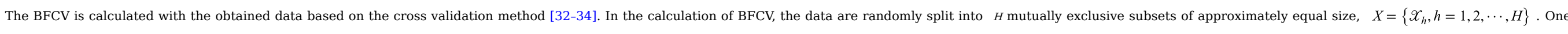

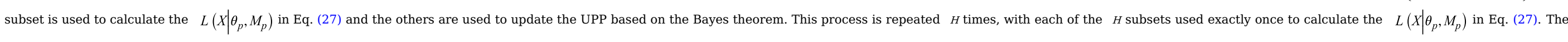
average of the $H$ is the corresponding marginal likelihood. Let $X / X_{h}$ denote all the data excluding $\mathscr{X}_{h}$. Then, Eqs. (28) and (26) can be reformulated as

$c v-B F_{p, q}=\frac{c v-p r\left(X \mid M_{p}\right)}{c v-p r\left(X \mid M_{q}\right)}, \quad p, q \in I_{c}$

where

and $H$ is called the fold of cross validation.

Annotations:

A1. please add a ' $p \in I_{-} c$,' at the end of the formula

A2. please add a ' $p \in I_{\_} c$,' at the end of the formula

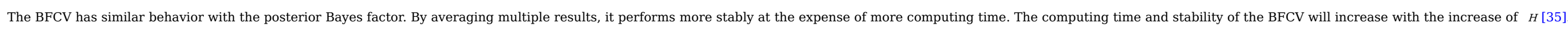

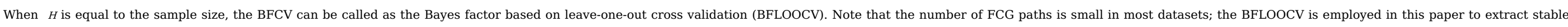
results. In addition, an easy-to-compute approximation [30] of the BFLOOCV can be utilized when the sample size is large.

\subsection{The implementation of the Bayesian model selection method}

\subsubsection{Candidate models}

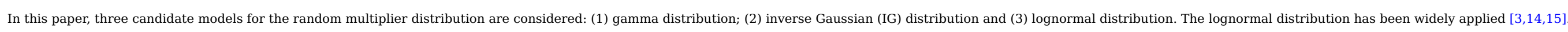

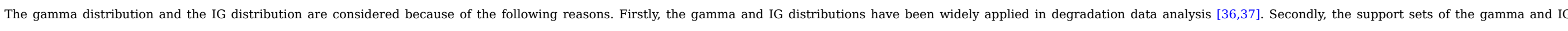

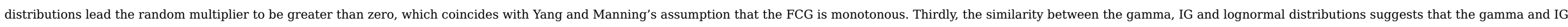
distributions are good potential alternatives for the lognormal [37].

Let $M_{1}, M_{2}$ and $M_{3}$ denote the gamma, IG and lognormal distributions, respectively. Their detailed PDFs are as follows:

$M_{1}$

$$
f_{M_{1}}(x \mid \alpha, \beta)=\frac{1}{\Gamma(\alpha) \cdot \beta^{\alpha}} x^{\alpha-1} \exp \left(-\frac{x}{\beta}\right), \alpha>0, \beta>0
$$


$f_{M_{2}}(x \mid \eta, \rho)=\left[\frac{\eta}{2 \pi x^{3}}\right]^{\frac{1}{2}} \exp \left[\frac{-\eta(x-\rho)^{2}}{2 \rho^{2} x}\right], \eta>0, \rho>0$

$M_{3}$

$f_{M_{3}}(x \mid \mu, \sigma)=\frac{1}{x \sigma \sqrt{2 \pi}} \exp \left[-\frac{(\ln x-\mu)^{2}}{2 \sigma^{2}}\right], \mu \in(-\infty,+\infty), \sigma>0$

\subsubsection{Model prior p(M)}

The model prior probabilities can be elicited from expert judgment or derived from the last Bayesian model selection analysis using historical data. When no information is available, uniform prior can be applied.

\subsubsection{Calculation of the Bayes factor}

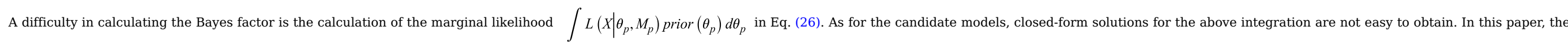

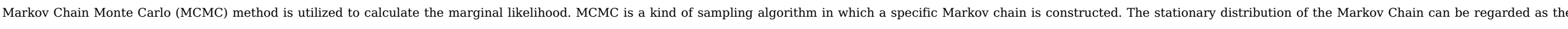

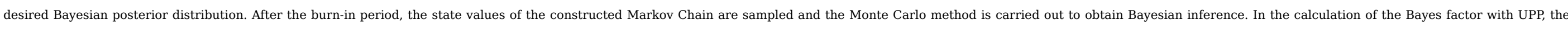

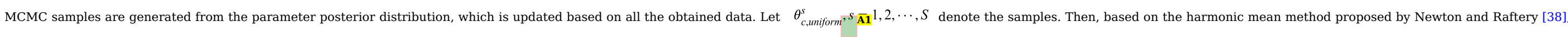
the corresponding marginal likelihood can be approximated as

$\operatorname{pr}\left(X \mid M_{c}\right) \approx\left[\frac{1}{S} \sum_{s=1}^{S} \frac{1}{L\left(X \mid \theta_{c, \text { uniform }}^{s}, M_{c}\right)}\right]^{-1} \operatorname{pr}\left(X \mid M_{c}\right) \approx\left[\frac{1}{S} \sum_{s=1}^{S} \frac{1}{L\left(X \mid \theta_{c, \text { uniform }}^{s}, M_{c}\right)}\right]^{-1}, c \in I_{c}$

Annotations:

A1. please add a formula 'c $\in$ I_c,' between the comma and the letter 's'

In the calculation of the posterior Bayes factor, the MCMC samples are generated from the parameter posterior distribution, which is also updated based on all the obtained data. Let corresponding marginal likelihood can be approximated as

$\operatorname{pr}\left(X \mid M_{c}\right) \approx \frac{1}{S} \sum_{s=1}^{S} L\left(X \mid \theta_{c, p o s p r i o r}^{s}, M_{c}\right) \operatorname{pr}\left(X \mid M_{c}\right) \approx \frac{1}{S} \sum_{s=1}^{S} L\left(X \mid \theta_{c, p o s p r i o r}^{s}, M_{c}\right), c \in I_{c}$

\section{Annotations:}

A1. please add a formula 'c $\in$ I_c,' between the comma and the letter 's'

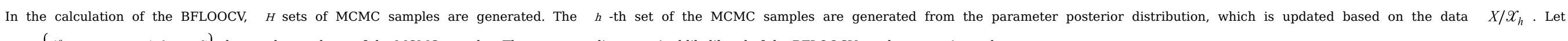
$\Theta_{c, h-C \text { Vprior }}=\left\{\theta_{c, h-C V \text { prior }}^{s}, s=1,2, \cdots, S\right\}$ denote the $h$-th set of the MCMC samples. The corresponding marginal likelihood of the BFLOOCV can be approximated as

$\operatorname{pr}\left(X \mid M_{c}\right)=\frac{1}{H} \sum_{h=1}^{H} \operatorname{pr}\left(X_{h} \mid M_{c}, X / X_{h}\right)$

where

$\operatorname{pr}\left(\mathscr{X}_{h} \mid M_{c}, X / X_{h}\right) \approx \frac{1}{S} \sum_{s=1}^{S} L\left(X_{h} \mid \theta_{c, h-C V p r i o r}^{s}, M_{c}\right) \quad \mathbf{A} 2$ 


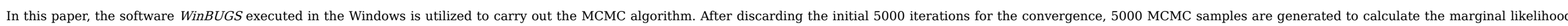
based on Eqs. (41)-(44). When the parameter space is unbounded, a normal distribution with a large variance ( $10^{6}$ in WinBUGS) is set to approximate the corresponding UPP as

$$
\text { prior }_{\text {uniform }}(\theta) \approx f_{\text {normal }}\left(\theta \mid \text { mean }=0 \text {, variance }=1 \times 10^{6}\right)
$$

The flowchart of the whole proposed method is shown in Fig. 1.

Estimate the parameters of the deterministic FCG
models by using first-step Least-Square estimation

\begin{tabular}{|c|} 
Assess four types of deterministic FCG models by using \\
adjusted- $R^{2}$
\end{tabular}

$\frac{\text { adjusted- }-R^{2}}{\downarrow}$ Generate samples for the estimation of the random

multiplier by using second-step Least-Square estimation

Calculate the Bayes factor for three candidate

distribution based on the generated samples

\begin{tabular}{|l|} 
Determine the distribution of the random multiplier by \\
using the Bayes model selection method
\end{tabular}

using the Bayes model selection method

Obtain the statistical inference of $\mathrm{FCG}$ rate based on the

most appropriate FCG model and the determined

distribution

Fig. 1 Flowchart of the proposed method.

\section{Simulation experiment}

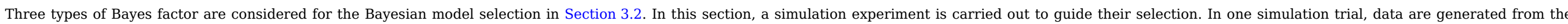

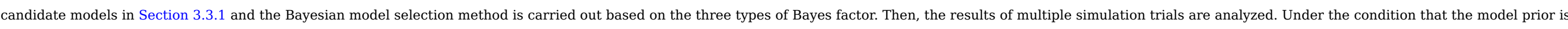

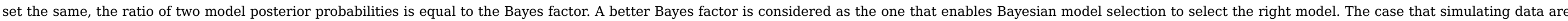
generated from a mixed distribution of candidate models is not studied in this paper.

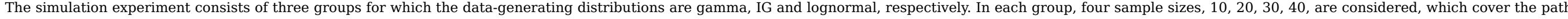

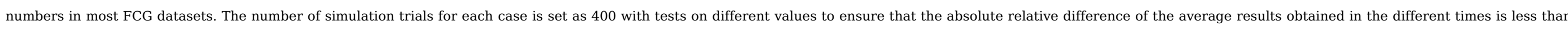
$5 \%$ for each group. The simulation setting is shown in Table 2 .

Table 2 Simulation setting

\begin{tabular}{|c|c|c|c|c|c|}
\hline \multirow[b]{2}{*}{ Group 1} & \multirow[t]{2}{*}{ Data-generating distribution } & \multicolumn{4}{|c|}{ Sample size } \\
\hline & & 10 & 20 & 30 & 40 \\
\hline Group 2 & IG & 10 & 20 & 30 & 40 \\
\hline Group 3 & lognormal & 10 & 20 & 30 & 40 \\
\hline
\end{tabular}

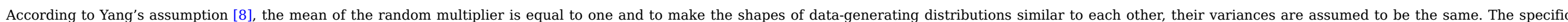
parameter values are shown in Table 3. 
Table 3 Parameter values of data-generating distributions.

\begin{tabular}{|c|c|c|c|}
\hline & Data-generating distribution & & Parameter value \\
\hline \multirow[t]{2}{*}{ Group 1} & \multirow[t]{2}{*}{ gamma } & $\alpha$ & 1 \\
\hline & & $\beta$ & 1 \\
\hline \multirow[t]{2}{*}{ Group 2} & \multirow[t]{2}{*}{ IG } & $\eta$ & 1 \\
\hline & & $\rho$ & 1 \\
\hline \multirow[t]{2}{*}{ Group 3} & \multirow[t]{2}{*}{ lognormal } & $\mu$ & $-\frac{\ln 2}{2}$ \\
\hline & & $\sigma$ & $\sqrt{\ln 2}$ \\
\hline
\end{tabular}

Three indicators are used to assess the performance of the three types of Bayes factor, which are the mean error (ME), the mean square error (MSE) and the proportion of the best (PB). The ME is defined as

$$
M E=\frac{1}{400} \sum_{t=1}^{400}\left(\frac{\sum_{p \in I_{c}, p \neq r i g h t} B F_{p, r i g h t}^{t}}{\sum_{p \in I_{c}} B F_{p, r i g h t}^{t}}\right)
$$

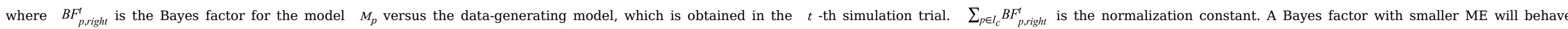
better in average sense.

The MSE is defined as

$$
M S E=\frac{1}{400} \sum_{t=1}^{400}\left(\frac{\sum_{p \in I_{c}, p \neq r i g h t} B F_{p, r i g h t}^{t}}{\sum_{p \in I_{c}} B F_{p, r i g h t}^{t}}\right)^{2}
$$

MSE is a comprehensive measure of bias and variance. A smaller MSE indicates better and more stable performance.

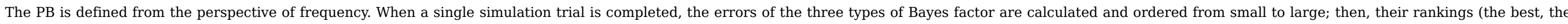

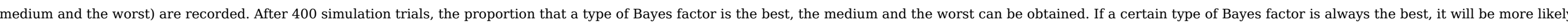
to be the best in the future.

The ME, MSE and PB for each group are shown in Figs. 2-4. 


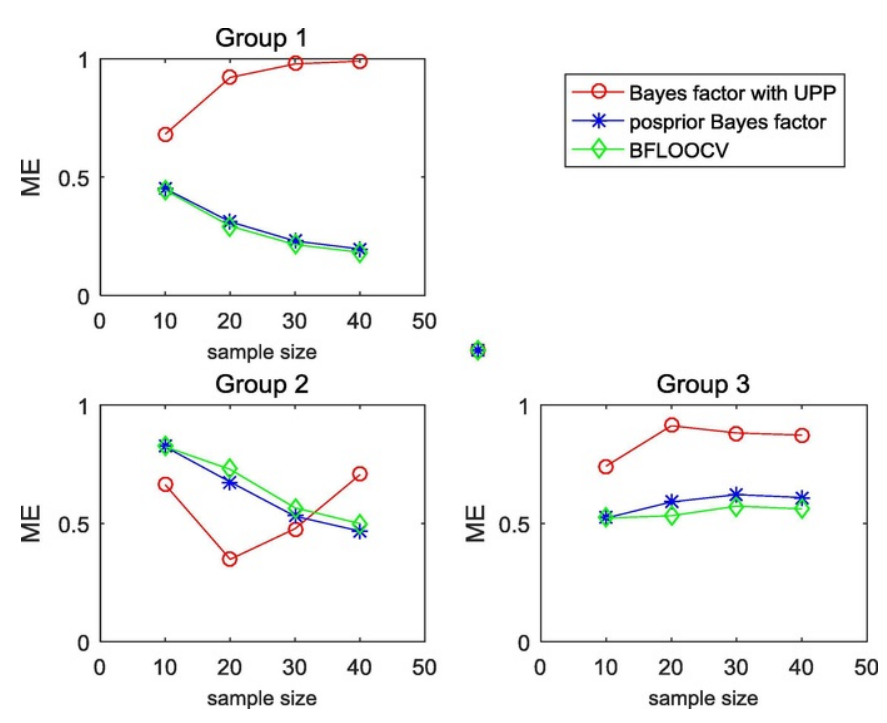

Fig. $2 \mathrm{ME}$ of the three types of Bayes factor.

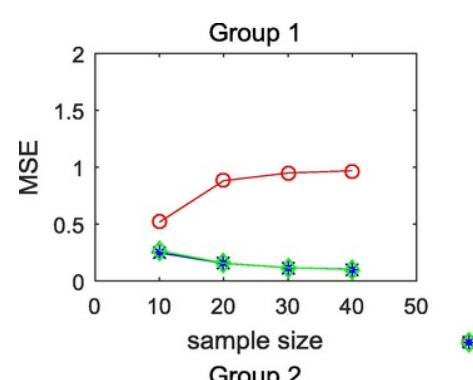

$$
\begin{aligned}
& -\bigcirc \text { - Bayes factor with UPP } \\
& * \text { - posprior Bayes factor } \\
& -\checkmark \text { BFLOOCV } \\
& \hline
\end{aligned}
$$
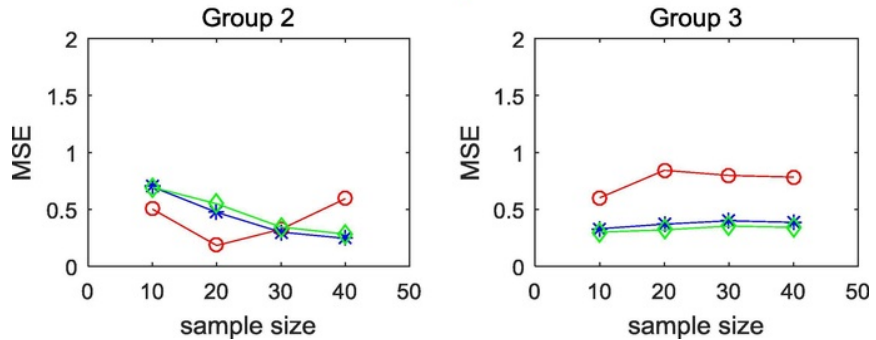

Fig. 3 MSE of the three types of Bayes factor. 


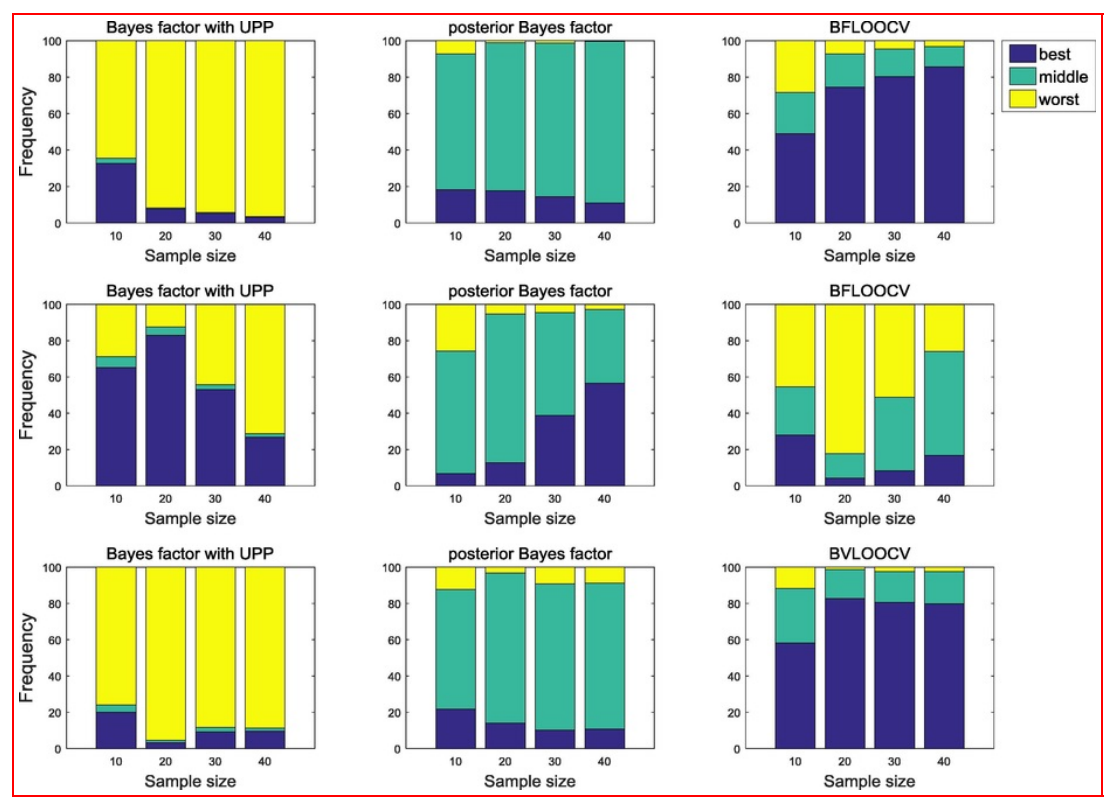

Fig. 4 PB of the three types of Bayes factor.

Based on the above results, some conclusions can be drawn as follows:

(1) The performance of the posterior Bayes factor and the BFLOOCV are similar from the perspective of ME and MSE, no matter what the data-generating distribution and sample size are.

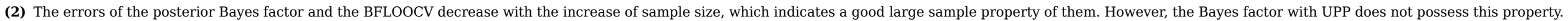

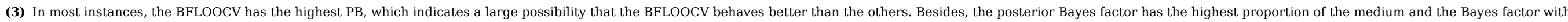
UPP has the highest proportion of the worst.

As a result, the BFLOOCV is selected in this paper to carry out the Bayesian model selection method.

\section{Case study}

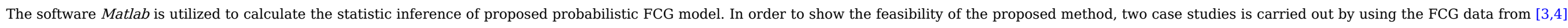
As for the FCG data, there are three main aspects that can affect the data quality: unit-to-unit variation, measurement error and sample size.

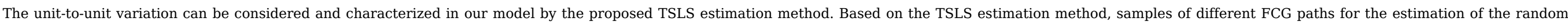
multiplier can be generated. Then, the random multiplier is modeled by a probabilistic distribution in consideration of unit-to-unit variation.

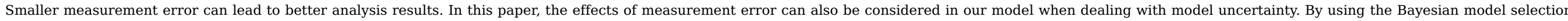

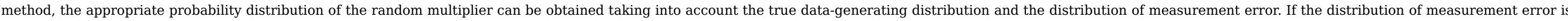
available, Bayesian filtering method [39] can be employed to estimate the true crack length, which hasn't been explored in this paper. We plan to investigate this issue in future work.

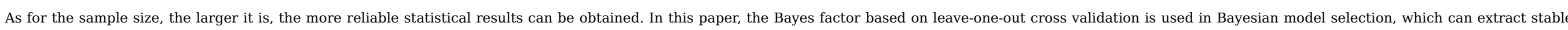

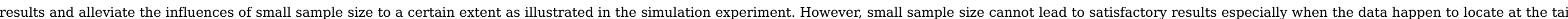
of the distribution. In fact, small sample size is a universal challenge and appropriate experimental sample size is problem-specific. The details of case studies are as follows: 


\subsection{Case 1: FCG dataset from wu and Ni's work [3]}

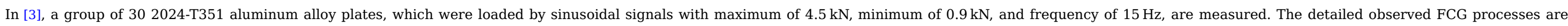
shown in Fig. 5

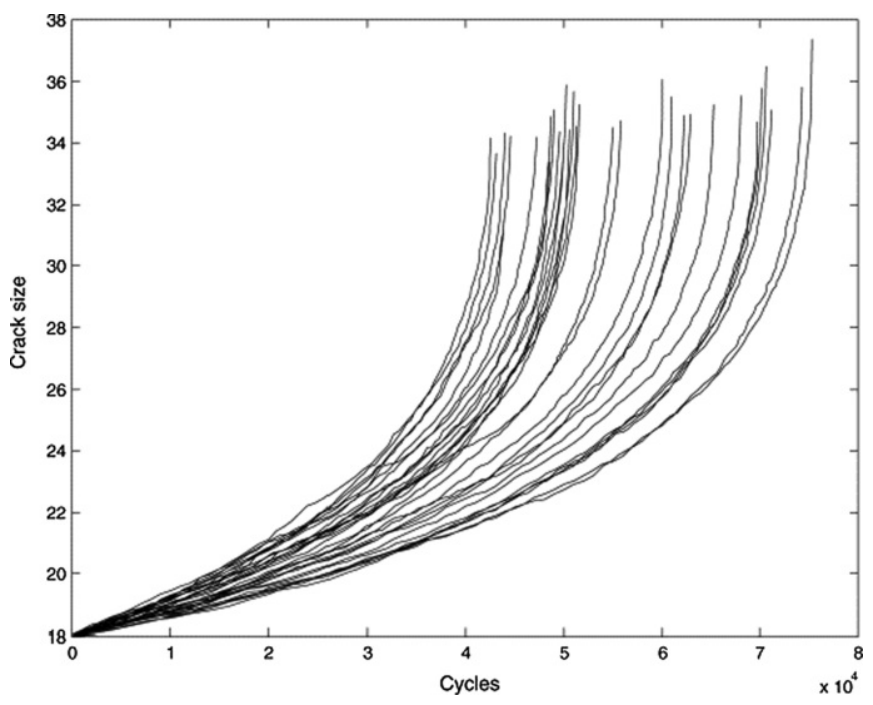

Fig. 5 FCG dataset from [3].

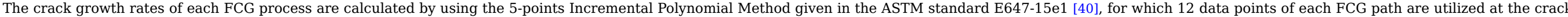

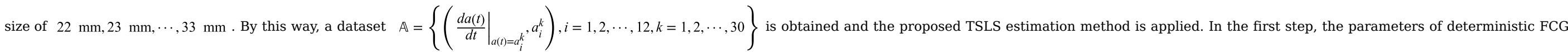
models are estimated, which are listed in Table 4. The corresponding fitting curves are plotted in Fig. 6.

Table 4 Parameter estimates of deterministic FCG models.

\section{Power function}

\begin{tabular}{l|l}
\hline$Q$ & $5.30 \mathrm{e}-14$ \\
\hline$b$ & 7.04 \\
\hline
\end{tabular}

polynomial function

\begin{tabular}{|l|l|}
\hline$p$ & $1.17 \mathrm{e}-2$ \\
\hline$q$ & $-1.01 \mathrm{e}-3$ \\
\hline$r$ & $0.02 \mathrm{e}-3$ \\
\hline
\end{tabular}

\begin{tabular}{|l|l|}
\hline$Q_{1}$ & $6.20 \mathrm{e}-8$ \\
\hline$b$ & 2.60 \\
\hline$Q_{2}$ & 4.80 \\
\hline
\end{tabular}




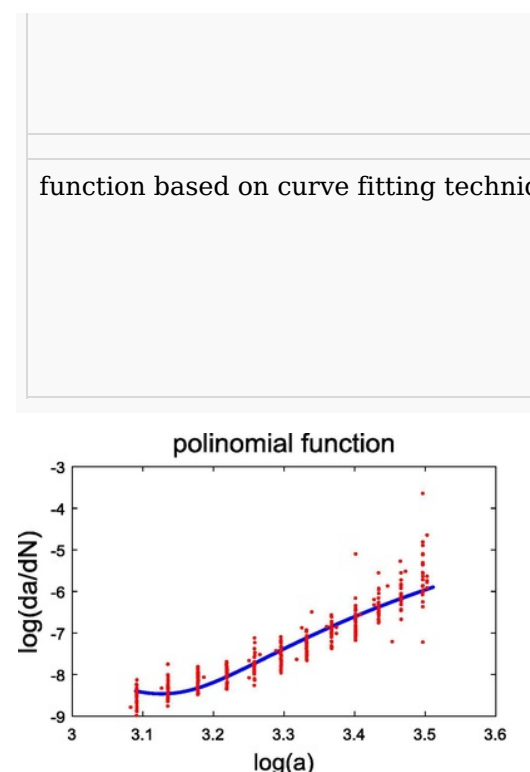

$Q_{3}$

0.81

$Q_{1}$

$244.26 \mathrm{e} 3$

\begin{tabular}{|l|l}
\hline 1 & -0.99 \\
\hline$b$ & $-7.56 \mathrm{e} 3$ \\
\hline$Q_{2}$ &
\end{tabular}

tional function
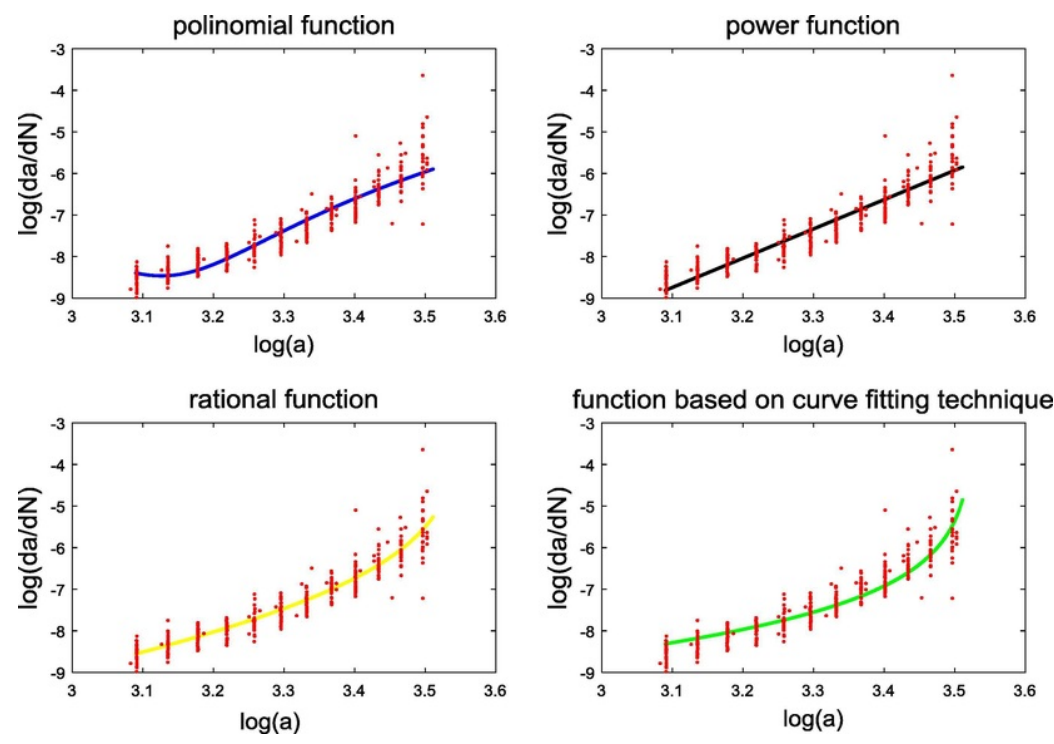

function based on curve fitting technique

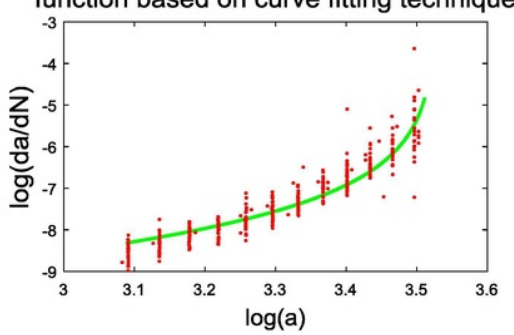

Fig. 6 Data points and corresponding fitting curves.

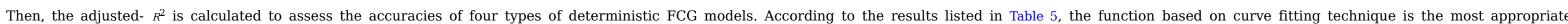
deterministic FCG model, which should be employed in the second step of the TSLS estimation.

Table 5 Adjusted- $R^{2}$ of four types of deterministic FCG models.

\begin{tabular}{|c|c|c|c|c|}
\hline Deterministic FCG model & Power function & Polynomial function & Rational function & \\
\hline Adjusted- $R^{2}$ & 0.8710 & 0.8758 & 0.8924 & 0.8925 \\
\hline
\end{tabular}

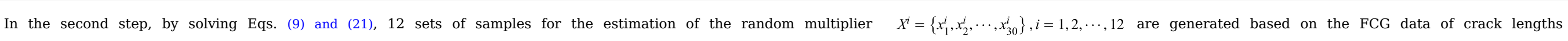

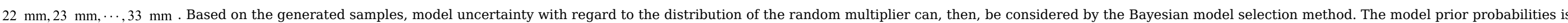
set to be uniformly distributed and the calculated Bayes factors of each candidate model are normalized and plotted in Fig. 7. 


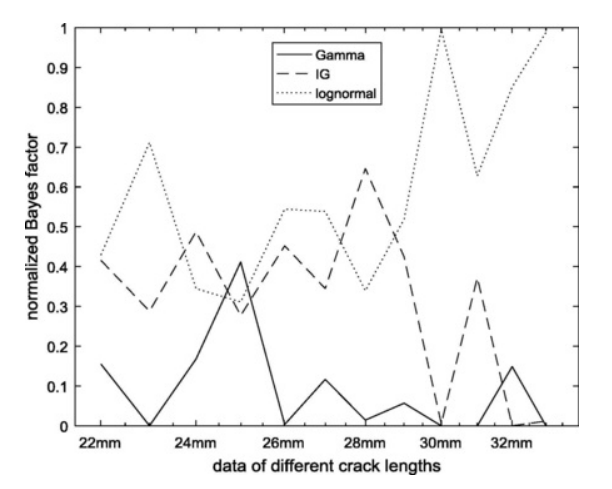

Fig. 7 The normalized Bayes factor for data of different crack lengths.

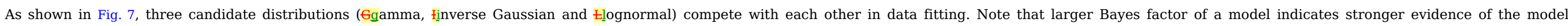

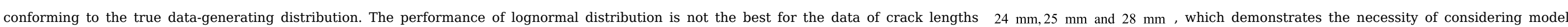

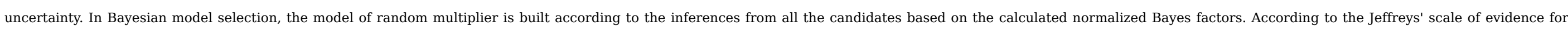

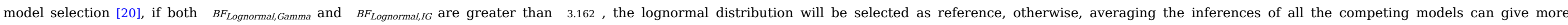

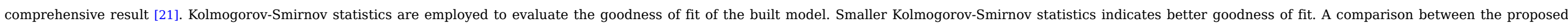
model and the Yang and Manning's model is provided in Fig. 8. It can be observed that the proposed model fit the data better than the Yang and Manning's model.

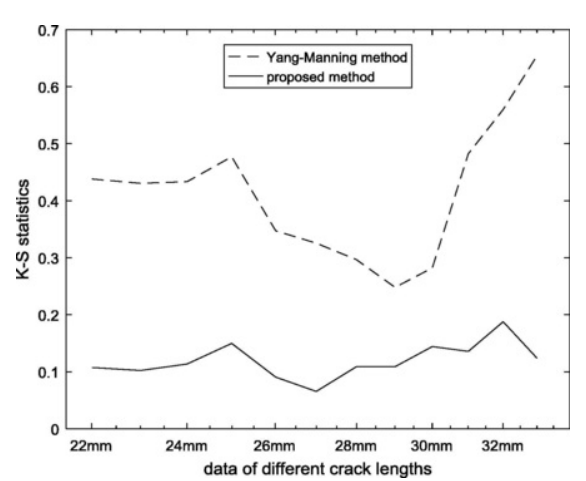

Fig. 8 The comparison of two methods for data of different crack lengths.

\subsection{Case 2: FCG dataset from Virker's work [4]}

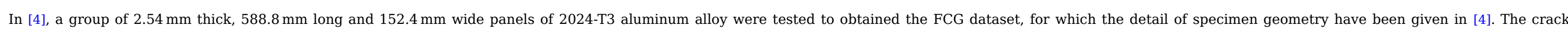

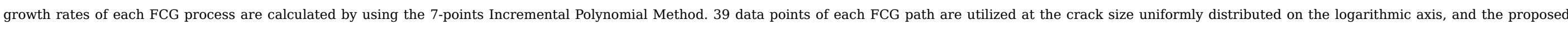
TSLS estimation is applied.

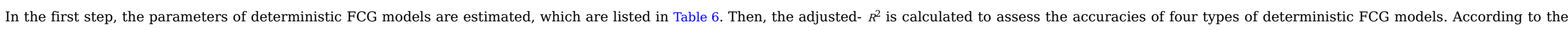
results listed in Table 7, the Polynomial function is the most appropriate deterministic FCG model, which should be employed in the second step of the TSLS estimation.

Table 6 Parameter estimates of deterministic FCG models. 


\begin{tabular}{|c|c|c|}
\hline & $b$ & 2.8699 \\
\hline \multirow[t]{3}{*}{ polynomial function } & $p$ & -38.8009 \\
\hline & $q$ & 3.4177 \\
\hline & $r$ & 0.4217 \\
\hline \multirow[t]{4}{*}{ rational function } & $Q_{1}$ & 0.0831 \\
\hline & $b$ & 3.1214 \\
\hline & $Q_{2}$ & 1.6165 \\
\hline & $Q_{3}$ & -0.4832 \\
\hline \multirow[t]{3}{*}{ function based on curve fitting technique } & $Q_{1}$ & 253.9450 \\
\hline & $b$ & -4.0506 \\
\hline & $Q_{2}$ & 0.0049 \\
\hline
\end{tabular}

Table 7 Adjusted- $R^{2}$ of four types of deterministic FCG models.

\begin{tabular}{|c|c|c|c|c|}
\hline Deterministic FCG model & Power function & Polynomial function & Rational function & Function based on curve fitting technique \\
\hline Adjusted- $R^{2}$ & 0.9064 & 0.9188 & 0.9067 & 0.9165 \\
\hline
\end{tabular}

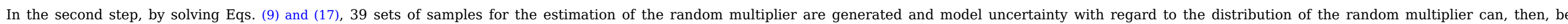

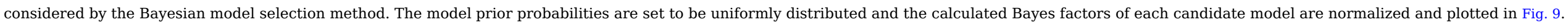

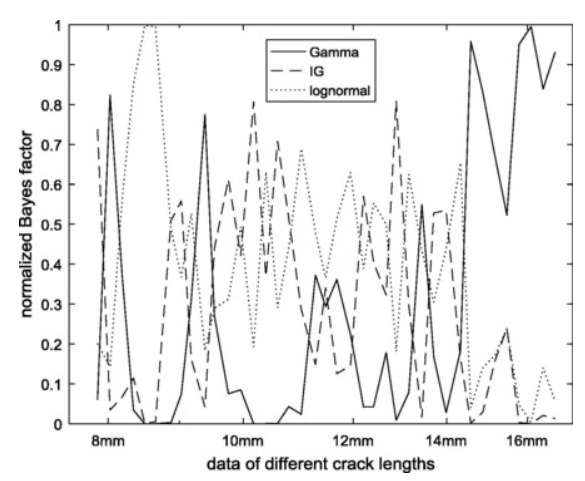

Fig. 9 The normalized Bayes factor for data of different crack lengths. 


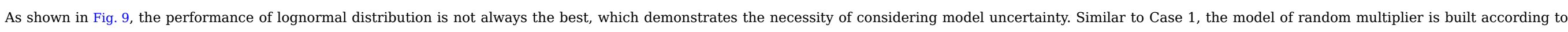

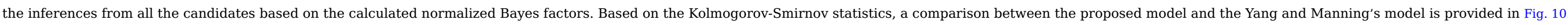
It can be observed that the proposed model fit the data better than the Yang and Manning's model.

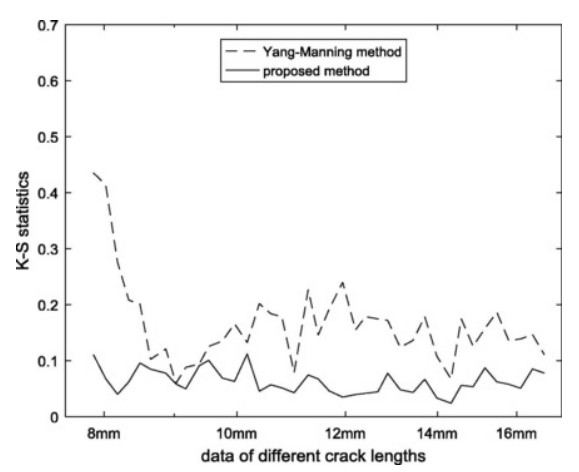

Fig. 10 The comparison of two methods for data of different crack lengths.

\section{Conclusion}

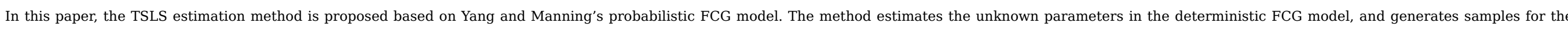

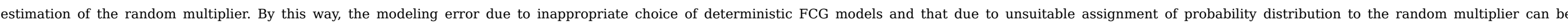

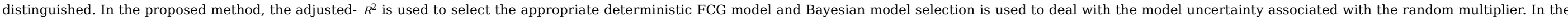

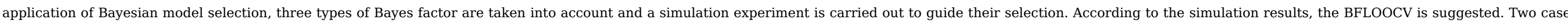
studies based on real FCG data are presented to illustrate the feasibility of the proposed method and the necessity of considering model uncertainty.

\section{Acknowledgement}

This work was supported by Science Challenge Project (No. TZ2018007), by the National Natural Science Foundation of China (Grant no. 51875016).

\section{References}

[1] P.C. Paris, M.P. Gomez and W.E. Anderson, A rational analytic theory of fatigue, Trend Eng 13, 1961, 9-14.

[2] J.M. Larsen, B.J. Schwartz and C.G. Annis, Jr, Cumulative Damage Fracture Mechanics Under Engine Spectra. Cumulative Damage Fracture Mechanics Under Engine Spectra, 1980.

[3] W. Wu and C. Ni, A study of stochastic fatigue crack growth modeling through experimental data, Probab Eng Mech 18 (2), 2003, $107-118$.

[4] D.A. Virkler, B. Hillberry and P. Goel, The statistical nature of fatigue crack propagation, J Eng Mater Technol 101 (2), 1979, 148-153.

[5] J. Yang and S. Manning, Stochastic crack growth analysis methodologies for metallic structures, Eng Fract Mech 37 (5), 1990, 1105-1124.

[6] W.-F. Wu, C. Shin and J.-J. Shen, Probabilistic analysis of fatigue crack propagation under random loading, J Pressure Vessel Technol 116 (2), $1994,216-225$.

[7] K. Sobczyk and B. Spencer, Jr, Random fatigue: from data to theory, 2012, Academic Press.

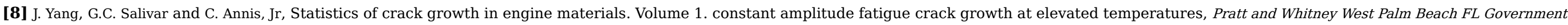
Products Div. 1982.

[9] I.T. Yu and C.L. Chang, Applying Bayesian model averaging for quantile estimation in accelerated life tests, IEEE Trans Reliab 61 (1), 2012 , 74-83. 
[10] K. Hamdia, et al., Assessment of computational fracture models using Bayesian method, Eng Fract Mech 205, 2019, 387-398.

[11] R. Zhang and S. Mahadevan, Model uncertainty and Bayesian updating in reliability-based inspection, Struct Saf 22 (2), 2000, 145-160.

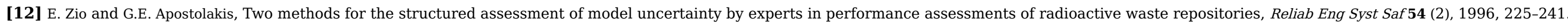

[13] I. Park, H.K. Amarchinta and R.V. Grandhi, A Bayesian approach for quantification of model uncertainty, Reliab Eng Syst Saf 95 (7), $2010,777-785$.

[14] G.S. Wang, Analysing the onset of multiple site damage at mechanical joints, Int J Fract 105 (3), 2000, 209-241.

[15] J.N. Yang and S.D. Manning, A simple second order approximation for stochastic crack growth analysis, Eng Fract Mech 53 (5), 1996, 677-686.

[16] H. Akaike, Information theory and an extension of the maximum likelihood principle, In: Inter. symp. on Information Theory vol. 1, 1973, 610-624.

[17] R.E. Kass and A.E. Raftery, Bayes factor, J Am Stat Assoc 90 (430), 1995, 773-795.

[18] L. Liu, et al., Model uncertainty in accelerated degradation testing analysis, IEEE Trans Reliab 66 (3), 2017, 603-615.

[19] I. Klugkist and H. Hoijtink, The Bayes factor for inequality and about equality constrained models, Comput Stat Data Anal 51 (12), 2007, 6367-6379.

[20] H. Jeffreys, The theory of probability, 1998, OUP Oxford.

[21] X. Guan, R. Jha and Y. Liu, Model selection, updating, and averaging for probabilistic fatigue damage prognosis, Struct Saf 33 (3), $2011,242-249$.

[22] Salimi H, Pourgol-Mohammad M, Kiad S. Assessment of Stochastic Fatigue Failures Based on Deterministic Functions.

[23] K.M. Hamdia, et al., Uncertainty quantification of the fracture properties of polymeric nanocomposites based on phase field modeling, Compos Struct 133, 2015, 1177-1190.

[24] J.N. Yang and R.C. Donath, Statistical crack propagation in fastener holes under spectrum loading, J Aircraft 20 (12), 1983, $1028-1032$.

[25] P. Paris and F. Erdogan, A critical analysis of crack propagation laws, J Basic Eng 85 (4), 1963, 528-533.

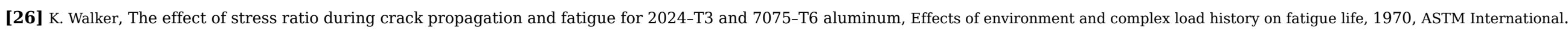

[27] R.G. Forman, V. Kearney and R. Engle, Numerical analysis of crack propagation in cyclic-loaded structures, J Basic Eng 89 (3), 1967, 459-463.

[28] D.C. Montgomery, G.C. Runger and N.F. Hubele, Engineering statistics, 2009, John Wiley \& Sons.

[29] M. Aitkin, Posterior Bayes Factors, J Roy Stat Soc: Ser B (Methodol) 53 (1), 1991, 111-142.

[30] A. Vehtari, A. Gelman and J. Gabry, Practical Bayesian model evaluation using leave-one-out cross-validation and WAIC, Stat Comput 27 (5), $2017,1413-1432$.

[31] A. Gelman, et al., Bayesian data analysis, 2013, Chapman and Hall/CRC.

[32] M. Stone, An asymptotic equivalence of choice of model by cross-validation and Akaike's criterion, J Roy Stat Soc Ser B (Methodol) $1977,44-47$.

[33] A. Vehtari and J. Lampinen, Bayesian model assessment and comparison using cross-validation predictive densities, Neural Comput 14 (10), $2002,2439-2468$.

[34] M.F. Badawy, et al., Hybrid nonlinear surrogate models for fracture behavior of polymeric nanocomposites, Probab Eng Mech 50, 2017 , 64-75.

[35] A. Celisse and S. Robin, Nonparametric density estimation by exact leave-p-out cross-validation, Comput Stat Data Anal 52 (5), $2008,2350-2368$.

[36] J. Lawless and M. Crowder, Covariates and random effects in a gamma process model with application to degradation and failure, Lifetime Data Anal 10 (3), $2004,213-227$.

[37] J.L. Folks and R.S. Chhikara, The inverse Gaussian distribution and its statistical application-a review, J Roy Stat Soc Ser B (Methodol) 40 (3), 1978, $263-289$. 
[39] S. Särkkä, Bayesian filtering and smoothing vol. 3, 2013, Cambridge University Press.

[40] Standard test method for measurement of fatigue crack growth rates. Astm; 2008.

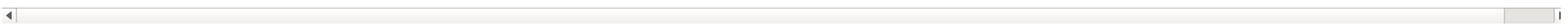

